\title{
Article \\ Adaptive Control for Energy Exchange with Probabilistic Interval Predictors in Isolated Microgrids
}

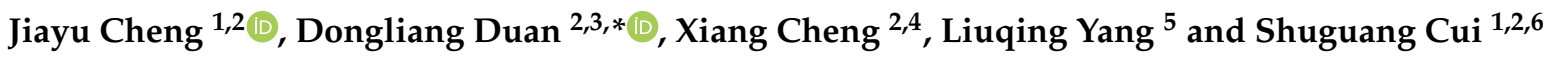 \\ 1 Shenzhen and Future Network of Intelligence Institute (FNii), The Chinese University of Hong Kong, \\ Shenzhen 518172, China; jiayucheng@link.cuhk.edu.cn (J.C.); shuguangcui@cuhk.edu.cn (S.C.) \\ 2 Shenzhen Research Institute of Big Data (SRIBD), Shenzhen 518172, China; xiangcheng@pku.edu.cn \\ 3 Department of Electrical and Computer Engineering, University of Wyoming, Laramie, WY 82071, USA \\ 4 State Key Laboratory of Advanced Optical Communication Systems and Networks, School of Electronics \\ Engineering and Computing Sciences, Peking University, Beijing 100080, China \\ 5 Department of Electrical and Computer Engineering, University of Minnesota, Minneapolis, MN 55455, USA \\ qingqing@umn.edu \\ 6 Department of Electrical and Computer Engineering, University of California at Davis, Davis, CA 95616, USA \\ * Correspondence: dduan@uwyo.edu
}

Citation: Cheng, J.; Duan, D.; Cheng, X.; Yang, L.; Cui, S. Adaptive Control for Energy Exchange with Probabilistic Interval Predictors in Isolated Microgrids. Energies 2021, 14, 375. https://doi.org/10.3390/ en14020375

Received: 3 November 2020 Accepted: 28 December 2020 Published: 12 January 2021

Publisher's Note: MDPI stays neutral with regard to jurisdictional clai$\mathrm{ms}$ in published maps and institutional affiliations.

Copyright: (C) 2021 by the authors. Licensee MDPI, Basel, Switzerland. This article is an open access article distributed under the terms and conditions of the Creative Commons Attribution (CC BY) license (https:// creativecommons.org/licenses/by/ $4.0 /)$.

\begin{abstract}
Stability and reliability are of the most important concern for isolated microgrid systems that have no support from the utility grid. Interval predictions are often applied to ensure the system stability of isolated microgrids as they cover more uncertainties and robust control can be achieved based on more sufficient information. In this paper, we propose a probabilistic microgrid energy exchange method based on the Model Predictive Control (MPC) approach to make better use of the prediction intervals so that the system stability and cost efficiency of isolated microgrids are improved simultaneously. Appropriate scenarios are selected from the predictions according to the evaluation of future trends and system capacity. In the meantime, a two-stage adaptive reserve strategy is adopted to further utilize the potential of interval predictions and maintain the system security adaptively. Reserves are determined at the optimization stage to prepare some extra capacity for the fluctuations in the renewable generation and load demand at the operation stage based on the aggressive and conservative level of the system, which is automatically updated at each step. The optimal dispatch problem is finally formulated using the mixed-integer linear programming model and the MPC is formulated as an optimization problem with a discount factor introduced to adjust the weights. Case studies show that the proposed method could effectively guarantee the stability of the system and improve economic performance.
\end{abstract}

Keywords: isolated microgrid system; interval predictions; microgrid energy exchange; model predictive control; reserve strategy; two-stage control

\section{Introduction \\ 1.1. Background}

The rapid development of the Internet of Things (IoT) technology enhances the intelligence in smart grid. Smart meters increase the measurement reporting rate in the grid to minute or even second level, and near real-time data communications with high throughput and low delay could be achieved with advanced technologies $[1,2]$. The integration of these advanced sensing and communication techniques greatly facilitates the integrated energy source management to improve the intelligence level and efficiency of the power grid [3].

Nowadays, Renewable Energy Sources (RES) such as solar and wind energies are increasingly integrated into the power grids as clean sources that are environmentally friendly $[4,5]$. They could also reduce generation cost and increase power diversification [6]. In many rural areas such as villages and countrysides, there are abundant RES and the 
buildings are sparse with many open areas. As a result, using RES as the major energy supply is quite plausible. Furthermore, the distances among different sites may be very large while the load demand at individual sites may be low due to the small population. Therefore, transmitting power from the main grid to the remote user side is costly. As a result, isolated microgrids receive more and more attention as a more cost-effective alternative [7].

Isolated microgrids are small power grids consisting of loads, RES, distributed dispatchable generators, and energy storage systems. They operate as independent power systems isolated from the main grid [8]. The penetration of RES in the isolated microgrids is usually very high and most of the power supply would be provided by RES generation. However, RES generation is volatile and hard to predict. In the meantime, there is no support from the main utility grid to absorb the fluctuations in isolated microgrids [9]. Therefore, it is very crucial for isolated microgrids to develop effective scheduling strategies to plan for the variations in advance, so that the power supply is ensured and the system stability is maintained [10-12]. Furthermore, the operation of microgrid systems can be more cost effective with appropriate planning and control [13-16].

\subsection{Related Work}

Several control methods have been reported in the literature to smooth the frequency fluctuations and improve the economic performance while ensuring the power supply's reliability and system stability for isolated microgrids. Most of these methods are based on single-point prediction [17-20]. They use time series analysis, model-based or datadriven approaches, advanced machine learning methods, and other hybrid methods to predict load consumption and renewable generation in the next period of time, and then obtain the optimal scheduling policy for the deterministic predicted scenario. The multiobjective energy management architecture is also studied to improve the stability and economy simultaneously. For example, a multi-objective Energy Management System (EMS) framework coupling unit commitment and optimal power flow is proposed in [21], and the compromise solution is obtained using the $\epsilon$-constraint method and the Fuzzy Satisfying criterion. However, the performance of such optimization methods is limited because it relies heavily on the accuracy of the single-point prediction, which is difficult to guarantee due to the high uncertainty of RES in isolated microgrids [22,23]. Though great efforts have been made to improve the prediction accuracy, errors are inevitable and may render the dispatch largely sub-optimal. Worse still, the accumulation of errors over time may lead to the failure of the entire optimization process and even cause serious violation of the system.

To deal with the highly volatile nature of the RES, some methods take extra considerations of the prediction error. On top of the deterministic optimization of the system energy management, they further compensate for the error discretely between each optimization stage, or directly take the uncertainty into consideration during optimization. In general, the former usually adopts a two-stage or hierarchical control strategy, and the latter mostly applies statistical methods or stochastic optimization methods. For instance, uncertainty was handled using a two-stage stochastic formulation in [24,25], where infeasibility from the first stage was compensated for at the second stage. In [26], the uncertainty associated with RES was modeled using the 2-Point Estimate Method (2PEM) for household demand management. Some works achieve the optimum by assuming that the uncertainties of RES and loads follow a certain distribution and can be modeled using some assumed Probability Distribution Functions (PDFs). For example, a Gaussian disturbance was introduced in [27] and non-Gaussian wind power uncertainties were studied in [28]. Then, stochastic control was applied. These methods could improve the system's capability to cope with fluctuations for isolated microgrids to some extent, but they are still developed based on deterministic forecasting. In the meantime, it is really challenging to further improve the accuracy of deterministic predictions due to the volatile nature of wind energy and the irregularity of solar energy. Therefore, these methods suffer from the unavoidable 
inaccuracy in RES predictions and might lead to increased instability for microgrids with high variability and uncertainty. In addition, the assumptions about the uncertainty distributions are not always appropriate and the inaccuracy in the distribution modeling could further compromise the performance.

The key in microgrid control hinges upon the uncertainties in the uncontrollable RES generation and load demand. The goal is to improve the robustness of the microgrid system with satisfactory economic benefits. For isolated microgrids, especially those with highly variable RES, the probabilistic prediction-based interval control optimization would be a better alternative. This category of methods does not require high accuracy deterministic parameter predictions that are difficult to achieve. Instead, interval predictions on the RES generation and load demand are used, whose accuracy is much easier to ensure. Intuitively, the predicted intervals provide more information compared with the point predictions used in deterministic approaches. They better capture the uncertainties and the trends of RES generation and loads in the future. With such information, one can choose to make relatively conservative responses to future changes to ensure the stability and reliability of isolated microgrids when the interval predictions indicate the RES generation tend to be deficient in covering the load demand. In addition, one can also adopt more aggressive strategies to achieve better economic benefits when the interval predictions indicate that the situation tends to improve in the future. Flexibility in the tradeoff between system stability and economic benefits becomes possible due to the rich information contained in the interval predictions.

In the literature, there are many methods that provide accurate interval prediction of RES generation and loads. However, the utilization of the intervals for system scheduling and dispatch is rather primitive. For example, fuzzy models were employed to predict intervals of an appropriate width and confidence level to cover certain uncertainties of the system in some works [29-33]. However, most of them did not discuss how to further make use of the predicted intervals in system scheduling [29-31]. In [32], a robust method was proposed for the subsequent scheduling. However, it only considered the worst cases indicated by the interval predictions and hence was overly conservative to achieve satisfactory economic performance. In [33], the confidence level of the intervals was used as an optional parameter with which the level of constraint satisfaction and operating cost can be selected by operators, but this method still could not facilitate the flexibility and the adaptivity in the tradeoff between the system stability and economic benefits. In [34], the interval uncertainties were modeled using an affine algorithm to generate modified intervals with soft bounds and then the optimal power flow solution was found using Particle Swarm Optimization (PSO). This helped contract the intervals and decrease the cost, but the joint PDFs and heuristic methods used led to high computational complexity. Therefore, efforts should be made to better utilize the interval predictions and improve energy management performance.

\subsection{Contributions}

To obtain the optimal scheduling of isolated microgrids with volatile RES and achieve a more effective utilization of the interval predictions, we have proposed a Model Predictive Control (MPC)-based hybrid method [35]. This method improves the economic performance of the microgrid system and strategy analysis shows that it can be interpreted as a "conditional" robust method. However, further studies indicate that system stability under this method could be compromised for isolated microgrids with high RES penetration, especially in extreme cases. In this paper, we come up with an adaptive probabilistic control method for energy exchange to further improve the system stability of isolated microgrids while ensuring the economical efficiency. The optimal microgrid dispatch problem is formulated using the Mixed-Integer Linear Programming (MILP) model and MPC is employed as the framework of the optimization problem [36,37]. A discount factor is introduced for each step of the MPC framework to adjust the weight so that the scheduling in the near future is given heavier weight to ensure stability $[38,39]$. During the 
optimization, appropriate scenarios are selected from the interval predictions according to the relationship among RES generation, load demand, and the capacity of the system. In the meantime, a two-stage elastic reserve strategy is adopted to further utilize the potential of the predicted intervals and maintain the system security adaptively. In this strategy, the possible trends of the situation and the state dynamics of the system are considered, based on which some auxiliary coefficients are added to automatically regulate the aggressive conservative level of the optimization process. Then, reserves are determined on the basis of the level to ensure the system capacity margin in case of potential fluctuations in RES generation and load at the operational stage. This promotes a more efficient use of system resources, reducing the cost and achieving the adaptive security of the system. A comparison with the previous method and robust method is conducted in different cases, and the effectiveness of our approach in improving system stability and economic performance is verified.

The major contributions of this paper are as follows:

1. An adaptive probabilistic energy management method based on the MPC framework and two-stage control is proposed to improve the utilization of the interval predictions and the dispatch performances in isolated microgrids with high penetrations of RES;

2. An adaptive reserve strategy is adopted to further exploit the information provided by the interval predictions and prepare for the possible variations in the future so that the system stability could be maintained in an adaptive manner;

3. The aggressive-conservative level of system is also introduced to guarantee certain system capacity and adjust the reserve strategy, where the system state and the future trends are evaluated to affect the system propensity on making decisions;

4. Simulation results show that the proposed method achieves good performances in terms of both system stability and economic efficiency, and it is capable of maintaining good performance even when the situation is extremely hostile.

\subsection{Outline}

The remainder of this paper is organized as follows. System model and operation requirements of a general isolated microgrid are discussed in Section 2. The adaptive reserve strategy is introduced in Section 3. Section 4 present the optimization formulation based on the MPC framework and the two-stage control method. The energy exchange strategy is also analyzed in Section 4. The case studies on a typical isolated microgrid system and the performance comparisons between our proposed method and others are shown in Section 5. Concluding remarks are presented in Section 6.

\section{System Model}

Remote community microgrids that isolated from the main grid are considered in this paper. The isolated microgrid system generally consists of load, RES generation, dispatchable generators, and energy storage units as Figure 1 shows. Figure 2 shows the scheme of a typical islanded microgrid considered in this paper. In general, the RES generation and load consumption are uncontrollable and should be predicted first. Then based on the prediction, the system needs to solve optimization problems to find economic dispatch for the storage units and dispatchable generators while ensuring the system stability.

The hierarchical control model has been widely used for microgrid control [40]. The hierarchical structure consists of three control levels: Primary, secondary, and tertiary. The primary control mainly handles stability maintenance of voltage and frequency and achieves autonomous operation. The secondary control is used for frequency and voltage restoration and helps compensate for the fluctuations in time. At the secondary control level, the sampling time is on the order of seconds, larger than that of the primary level. The tertiary control has the longest sampling time from minutes to hours. At this level, economical concerns are considered and optimal operation is studied in the microgrids. 


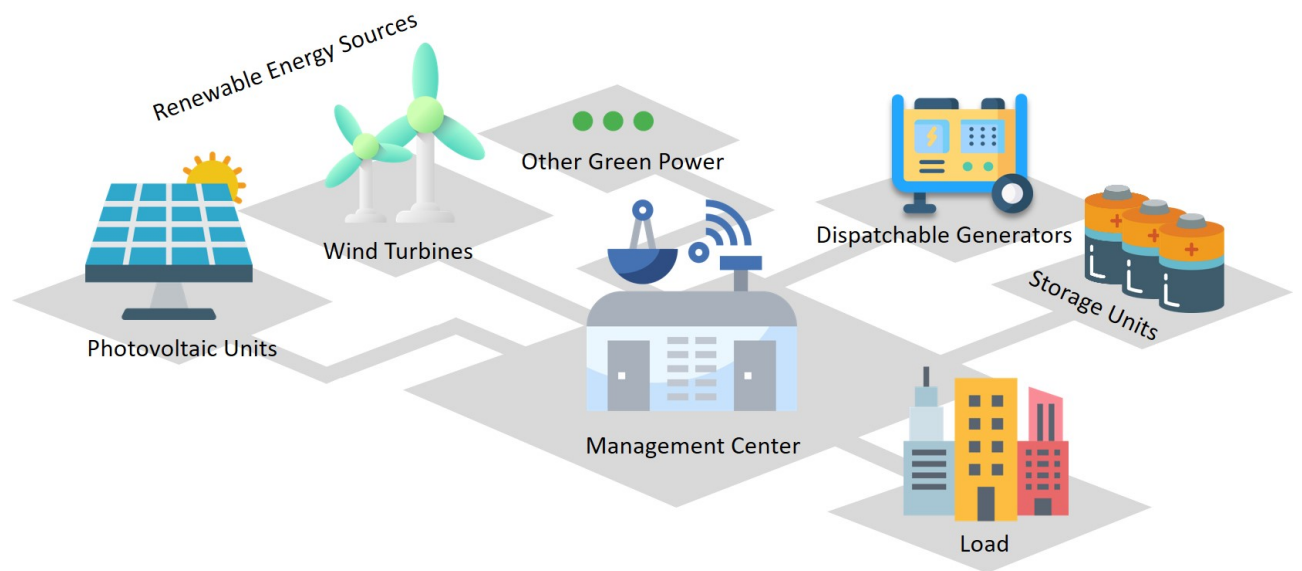

Figure 1. The system model of general isolated microgrids.

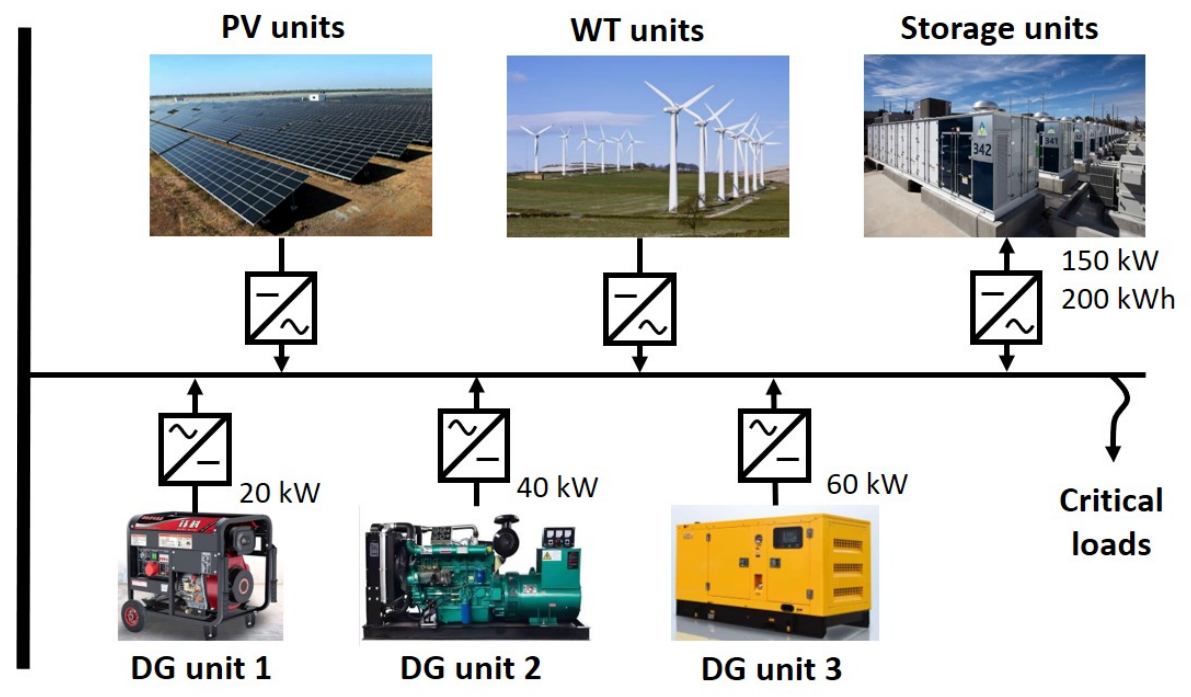

Figure 2. Scheme of a typical isolated microgrid including photovoltaic (PV) units, wind turbine (WT) units, storage units, and three different sets of diesel generators (DGs). The case is also studied in simulations.

This paper focuses on the optimal dispatch problem at the tertiary control level, where the supply and demand relationship, the operation limits, and the objectives concerning economy and robustness are the main issues discussed to find optimal scheduling of operating power for dispatchable devices in microgrids [17,41]. Therefore, no particular discussions on primary and secondary control are made in this paper. Noting that the time-scale of the primary and secondary control is far less than that at the tertiary level, in this paper we assume that they are conducted appropriately by some existing approaches.

In this section, we discuss the model of each type of components in the general microgrid. The device dynamics and some operation requirements are also considered. In the model, the long-term control problem is handled discretely with small time interval $\Delta t$, and the power of components is considered constant at each time interval [42].

\subsection{Interval Predictions}

RES generators are uncontrollable and non-dispatchable elements in microgrid systems. Loads generally include critical loads that must be met and controllable loads that can be reduced if necessary. Here, we assume all the loads in the isolated microgrid are critical and non-dispatchable. To meet the load demand and find the optimal scheduling 
policy of the system with low cost and high reliability, the value of load power and RES generation power of the future should be known. However, the intermittent and volatile nature of RES makes it really challenging to obtain a high accuracy in their generation forecasting as required to ensure the strict load and generation balanced of microgrids. As a result, in this paper, we try to use interval predictions rather than point predictions as the information to design the scheduling strategy. When the predictions are given in the form of intervals, they are no longer sensitive to small errors since the intervals cover a range rather than a single value. Hence it is much easier to obtain good interval predictors. Furthermore, intervals provide more information about possible values and trends in the future, based on which the system can be better prepared to maintain stability.

In this paper, the predictions in the form of intervals are described as the interval constraints:

$$
\begin{aligned}
P_{l}[k] & \in\left[\underline{P_{l}}[k], \overline{P_{l}}[k]\right], \\
P_{\text {res }, m}[k] & \in\left[\underline{P_{r e s, m}}[k], \overline{P_{r e s, m}}[k]\right],
\end{aligned}
$$

where $k$ is the discrete-time index, $P_{l}$ and $P_{r e s, m}$ are the total load power and the renewable generation power respectively with $m$ representing different type of RES such as photovoltaic power and wind power; the lower and upper boundaries of the prediction intervals are denoted by the underline and the overline of power variables, respectively. Interval predictions with good accuracy level could be provided by many existing works. For example, fuzzy interval models can be used to forecast intervals of RES generation and load power with appropriate width and coverage probability $[29,32]$. The interval predictions with $90 \%$ of confidence level have about $3.67 \%$ maximum relative error in photovoltaic power generation, $8.85 \%$ maximum relative error in wind power generation, and $4.53 \%$ maximum relative error in load power in [29]. We will see later that this level of accuracy is sufficient to implement our proposed algorithm.

\subsection{Dispatchable Generators}

Dispatchable generators are energy sources that can be controlled flexibly according to the dispatch and they usually employ non-renewable energy sources including petroleum, diesel, natural gas, and so on. These generators are available almost anywhere and can respond to demand quickly. Hence, they are always chosen to serve as a buffer between renewable power generation and load demand in microgrids with RES. Though dispatchable generators could meet the demand more flexibly, the usage of them should also be carefully planned to avoid violations and unnecessary wastage. There are some limits on their working range and operating behavior.

A. The working range:

Generally, the operating range of generators can be expressed as:

$$
\delta_{g, i} P_{g, i}^{\min } \leq P_{g, i} \leq \delta_{g, i} P_{g, i}^{\max },
$$

where $i=1,2, \ldots, N_{g}$ with $N_{g}$ representing the number of generators. $P_{g, i}$ denotes the operating power of the $i$ th generator, and $P_{g, i}^{\min }$ and $P_{g, i}^{\max }$ are the minimum and maximum power limits, respectively. $\delta_{g, i}$ is the introduced auxiliary binary variable used to indicate the ON/OFF state of the dispatchable generators:

$$
\delta_{g, i}=\left\{\begin{array}{l}
1, \text { ON state, } \\
0, \text { OFF state } .
\end{array}\right.
$$


B. The operating behavior:

It usually costs a lot to switch the state of generators. Thus, the startup and shutdown behavior of the generators is also modeled to simplify the calculation of the corresponding operating cost in the cost function. The following constraints must be satisfied:

$$
\begin{aligned}
& \left\{\begin{array}{l}
S U_{i}[k]=K_{i}^{S U} \cdot\left(\delta_{g, i}[k]-\delta_{g, i}[k-1]\right) \\
S D_{i}[k]=K_{i}^{S D} \cdot\left(\delta_{g, i}[k-1]-\delta_{g, i}[k]\right) \\
S U_{i}[k] \geq 0 \\
S D_{i}[k] \geq 0,
\end{array}\right. \\
& -R_{i}^{\max } \leq P_{g, i}[k+1]-P_{g, i}[k] \leq R_{i}^{\max },
\end{aligned}
$$

where $S U_{i}[k]$ and $S D_{i}[k]$ are the startup and shutdown costs of the $i$ th generator at time $k$, respectively; $K_{i}^{S U}$ and $K_{i}^{S D}$ are the corresponding coefficients representing the cost of a single switch with respect to the equipment specifications. The operating cost could be calculated by comparing the generation state at the current instance with that at the previous instance. $R_{i}^{\max }$ represents the maximum ramp up and ramp down rates. The inequality (5) limits the maximum changes of the running power of the $i$ th generator within a time interval $\Delta t$.

\subsection{Energy Storage Units}

A large number of non-dispatchable generators connected to the microgrid system may cause power fluctuations, and the trends in RES are volatile and hard to predict. Energy storage units play a significant role in the system to mitigate the effects caused by fluctuations and act as a buffer between generation and demand. Moreover, energy storage units could quickly respond to demand which makes them very suitable to compensate for prediction errors and disturbances.

The charging and discharging behavior of storage units and the operating constraints will be discussed in detail here. The storage dynamics of energy storage units is usually described using discrete-time models as follows [17]:

$$
W_{s}[k+1]=W_{s}[k]\left(1-\sigma_{s}\right)+\left(\eta \cdot P_{s}^{c}[k]-\frac{1}{\eta} \cdot P_{s}^{d i s c}[k]\right) .
$$

In this equation, $W_{s}[k]$ denotes the total stored energy of the storage units at time $k$, and it is bounded with $W_{s}^{\text {min }} \leq W_{s} \leq W_{s}^{\max } ; P_{s}^{c}$ and $P_{s}^{\text {disc }}$ are the charging and discharging power, respectively. $\sigma_{s}$ is the energy loss rate related to the stored energy degradation with $0<\sigma_{s}<1 . \eta$ is the charging and discharging efficiency with $0<\eta<1$.

Charging and discharging phases need to be considered separately [43]. Two auxiliary binary variables are introduced to represent the charging and discharging state of energy storage units, with $\delta_{c}=1, \delta_{\text {disc }}=0$ indicating the charging state and $\delta_{c}=0, \delta_{\text {disc }}=1$ indicating the discharging state. It is noted that these two variables are mutually-exclusive with the constraint:

$$
\delta_{c}+\delta_{\text {disc }} \leq 1 .
$$

Thus, the operating range of storage units in general is:

$$
P_{s}^{\min } \leq \delta_{c} \cdot P_{s}^{c}-\delta_{d i s c} \cdot P_{s}^{d i s c} \leq P_{s}^{\max } .
$$

\subsection{Energy Balance}

One of the key in mcirogrid energy management is maintaining the power balance between energy sources, storage units, and load. Since power is considered constant at 
each time interval $k$ and energy exchange problem is studied in this paper, the energy balance constraint is modeled instead:

$$
\sum_{m}\left(P_{r e s, m}[k] \Delta t\right)+\sum_{i=1}^{N_{g}}\left(P_{g, i}[k] \Delta t\right)=P_{l}[k] \Delta t+\left(P_{s}^{c}[k]-P_{s}^{d i s c}[k]\right) \Delta t .
$$

In the optimization, this balance condition is a constraint that must be satisfied and local controllers will help track the reference power at a lower control level in operation. At the operation stage, however, it may not hold with the dispatching solutions since the prediction is not exactly accurate and there are differences between the scenarios the optimizer selected and the actual situations. Then the load demand might not be met and violations occur. That is why the compensation should be considered at the operation stage so as to further handle the variations and that reservations are carefully planned.

\section{The Adaptive Reserve Strategy}

In some extreme cases, when the RES generation is extremely volatile and the prediction is not sufficiently accurate, the system stability and economic performance of general control methods may be hard to guarantee. To enhance the system stability and prepare more for the unknown variations, an adaptive reserve strategy is proposed in this paper.

\subsection{The Adaptive Reserves}

To make the system better prepared for future variations, we introduce some reserve variables in the models of energy storage units and dispatchable generators. These variables are assigned values in the optimization based on the comprehensive assessment of the system's capacity and future trends, and will be utilized at the operation stage if necessary. In other words, they are additional safeguards to compensate for deviations. Therefore, this part of capacity cannot be used at the scheduling stage and some constraints should be modified.

The reserve variable $P_{g, i}^{u r}$ is introduced into the model of dispatchable generators denoting an upward reserve power of $i$ th generator for generating, and constraint (2) and (5) are transformed using the big-M method [44] as follows:

$$
\begin{gathered}
\left\{\begin{array}{l}
P_{g, i}^{\min } \leq P_{g, i} \leq P_{g, i}^{\max }-P_{g, i}^{u r} \\
\delta_{g, i} \cdot P_{g, i}^{\min } \leq P_{g, i} \leq \delta_{g, i} \cdot P_{g, i}^{\max },
\end{array}\right. \\
-R_{i}^{\max } \leq P_{g, i}[k+1]-P_{g, i}[k] \leq R_{i}^{\max }-P_{g, i}^{u r}[k] .
\end{gathered}
$$

In the storage model we also add two reserve variables $P_{s}^{u r}$ and $P_{s}^{d r}$ which denote upward reserve power for charging and downward reserve power for discharging of the energy storage units. Hence, (7) and (8) are modified as:

$$
\left\{\begin{array}{l}
P_{s}^{\min } \leq P_{s}^{c} \leq P_{s}^{\max }-P_{s}^{\text {ur }} \\
\delta_{c} \cdot P_{s}^{\min } \leq P_{s}^{c} \leq \delta_{c} \cdot P_{s}^{\text {max }} \\
P_{s}^{\min } \leq P_{s}^{\text {disc }} \leq P_{s}^{\max }-P_{s}^{d r} \\
\delta_{\text {disc }} \cdot P_{s}^{\text {min }} \leq P_{s}^{\text {disc }} \leq \delta_{\text {disc }} \cdot P_{s}^{\text {max }}
\end{array}\right.
$$

where the big-M method is also adopted to avoid the bilinear representation.

In addition, the following constraints should be satisfied by these three reserve variables:

$$
\left\{\begin{array}{l}
0 \leq P_{s}^{u r} \leq \Delta P_{e}^{\max _{1}} \\
0 \leq P_{s}^{d r}+\sum_{i} P_{g, i}^{u r} \leq \Delta P_{e}^{\max _{2}}
\end{array}\right.
$$


with

$$
\left\{\begin{array}{l}
\Delta P_{e}^{\max _{1}}=\sum_{m}\left(\overline{P_{\text {res }, m}}-P_{\text {res }, m}\right)+\left(P_{l}-\underline{P_{l}}\right) \\
\Delta P_{e}^{\text {max }_{2}}=\sum_{m}\left(P_{\text {res }, m}-\underline{P_{r e s, m}}\right)+\left(\overline{P_{l}}-P_{l}\right),
\end{array}\right.
$$

where $\Delta P_{e}^{\max _{1}}$ and $\Delta P_{e}^{\max _{2}}$ denote the maximum power error between the actual situation and the selected scenarios in optimization in the conditional best and worse cases, respectively.

\subsection{The Adaptive Reserve Strategy}

To enhance the stability of the microgrid system and make use of the reserves more efficiently and sophisticated, an adaptive reserve strategy is proposed to adjust the reservation level determined in the optimization and the system behavior tendency in the operation.

Certain system capacity is reserved during the optimization process when selecting appropriate scenarios and scheduling the corresponding dispatches, with which the system is better prepared for the undesirable variations in the future. This part of capacity cannot be used during scheduling, but later in the operation, variations will be handled by the hierarchical control system and compensated using the pre-reserved capacity. This effectively ensures system stability. Moreover, these reserves are determined in optimization with the penalty term (22) and adjusted at each time instance, enhancing the flexibility of reservation and further improving system stability.

Furthermore, the aggressive level of the system is not fixed. We introduce two coefficients $K_{r, \text { eur }}$ and $K_{r, e d r}$ in the reserve cost to achieve the automatic adjustment of the aggressive and conservative level of system, which would influence the reservation degree and control policy. These two factors are set as auxiliary variables that are sensitive to system state and the possible trends of RES generation and load demand presented as follows:

$$
\left\{\begin{array}{l}
K_{r, \text { eur }}=\lambda_{1} \cdot\left(\Delta P_{h}-\mu_{1} \cdot \Delta P_{f}\right)+\kappa_{1} \\
K_{r, \text { edr }}=\lambda_{2} \cdot\left(\Delta P_{h}-\mu_{2} \cdot \Delta P_{f}\right)+\kappa_{2}
\end{array},\right.
$$

where $\Delta P_{h}$ is the average deviation in the system capacity between the selected scenarios and the actual operation in the past, indicating the situation of the system capacity at present. The system would try to restore it to the appropriate level based on the evaluation. $\Delta P_{f}$ shows the degree of capacity change we need to deal with in the future, which can be roughly represented by the average power difference between the renewable generation and load demand in future. The system would become more conservative if the situation in future is not good and prepare more for the demand at the cost of economic benefits. They can be formulated as follows with $r$ and $s$ denoting the number of steps taken into account:

$$
\left\{\begin{array}{l}
\Delta P_{h}=\frac{1}{r} \sum_{t=k-r}^{k}\left(\sum_{m}\left(P_{r e s, m}^{a c t}[t]-P_{r e s, m}^{o p}[t]\right)-\left(P_{l}^{a c t}[t]-P_{l}^{o p}[t]\right)\right) \\
\Delta P_{f}=\frac{1}{2 s} \sum_{t=k+1}^{k+s+1}\left(\sum_{m}\left(\overline{P_{r e s, m}[t]}+\underline{P_{r e s, m}[t]}\right)-\left(\overline{P_{l}[t]}+\underline{P_{l}[t]}\right)\right)
\end{array} .\right.
$$

Many groups of values will be assigned to the parameters in Equation (15) depending on the situation and trend of system capacity, which transforms the formulations into piece-wise linear functions. Based on them the aggressive-conservative level of the system is linked to the system state and the future situation of uncontrollable components. On the one hand, the two factors $K_{r, \text { eur }}$ and $K_{r, e d r}$ are determined according to the evaluation of the history and future conditions. On the other hand, these two factors would affect the dispatch policy obtained at the optimization stage. As a result, the aggressive and conservative level of the microgrid system is adaptively adjusted, where system stability is ensured when the situation is hostile and the system capacity is poor, and cost-effectiveness is achieved when everything is good.

The reserved capacity can be used at the operation stage to compensate for the possible variations, and the actual compensation power should be within the range set by the reserve decisions: 


$$
\left\{\begin{array}{l}
-P_{s}^{d r} \leq \Delta P_{s}^{c o m} \leq P_{s}^{u r} \\
0 \leq \Delta P_{g, i}^{c o m} \leq P_{g, i}^{u r} .
\end{array}\right.
$$

Here, the actual compensation power of energy storage units $\Delta P_{s}^{c o m}$ can be either positive or negative, while that of the $i$ th dispatchable generator $\Delta P_{g, i}^{c o m}$ can only take a non-negative value since dispatchable generators participate only when additional power generation is needed. The secondary control plays important roles in the corresponding operation and adjustment, and a simple but effective algorithm is adopted to regulate the compensation operation and further improve the economical efficiency. This has been represented in our previous work [35].

\section{Optimization and Control Method}

In this paper, the MPC method is used for optimization and a two-stage control method using an adaptive reserve strategy is proposed to solve the optimal dispatch problem and improve the stability of the system under variations. The strategy of energy exchange is also analyzed in this section.

\subsection{The MPC Framework}

Microgrid energy management is a continuous and long-term problem, and the optimal scheduling of a microgrid within the duration of one day to one year is usually taken into account. To ensure the accuracy of predictions and avoid the accumulation of disturbances, the problem is usually divided into many short-term control issues with small time intervals solved one by one. There are various approaches solving the microgrid power management reported in the literature. Some of the classical algorithms include mathematical programming [45,46], dynamic programming [47], and rule-based methods [48]. Other emerging methods are mostly developed based on heuristics [49,50], multi-agent system [51], stochastic and robust programming [52,53], and artificial intelligence techniques [54,55].

However, these methods usually focus only on the scheduling in the short term, and repeat the optimization operation in the next term to support the long-term dispatch. Nonetheless, the long-term trends should not be ignored since short-term optimization merely considers the present and cannot grasp the undesirable changes, which possibly leads to serious losses. MPC is a remarkable method that takes both the short-term control and the long-term trends into consideration $[36,56]$. Therefore, it helps improve the system reliability and it is particularly suitable for the dispatch problem in isolated microgrids. Moreover, the feedback mechanism is introduced in MPC to make the system more robust against uncertainty. System dynamics and the operation constraints could also be handled effectively in the MPC formulation. Existing work has validated the superior performance and effectiveness of MPC-based control methods when compared with other methods [57].

In the MPC framework, the time duration of the long-term optimal control problem $T$ is divided into many steps using the time step $\Delta t$, and the total number of control steps is $N$ with $T=N \Delta t$. At time step $k \in 1, \ldots, N$, the optimization problem from step $k$ to step $(k+M-1)$ considering future trends is solved and the optimal policy $\left\{u_{k}, \ldots, u_{k+M-1}\right\}$ for the $\mathrm{M}$ steps is found. However later, the system only executes the first action $u_{k}$ and adjust the components to respond to variations. Next at time step $(k+1)$, the predictions and the system state is updated, based on which a new optimization problem with the moving horizon from step $(k+1)$ to step $(k+M)$ is solved, and a new control policy $\left\{u_{k+1}, \ldots, u_{k+M}\right\}$ is obtained recursively. The optimization result is typically not globally optimal but the possible situations in the future are properly assessed when making the current policy, so that the system is better prepared for future events and more robust.

\subsection{The MPC-Based Optimization Problem}

The optimal dispatch problem is basically to minimize the total cost while ensuring the stability of microgrid system. The MPC-based optimization problem for isolated microgirds using interval predictions could be finally represented as below: 


$$
\begin{aligned}
& \operatorname{minimize} \sum_{k=t+1}^{t+M} \alpha^{k-t}\left[C_{o m}[k]+C_{g}[k]+C_{s}[k]+C_{r}[k]\right] \\
& \text { subject to (1), (4), (6), (9)-(14). }
\end{aligned}
$$

The objective consists of four terms including the operation and maintenance $\operatorname{cost} C_{o m}$ as given in (19), the energy production $\operatorname{cost} C_{g}$ as given in (20), the startup and shutdown $\operatorname{cost} C_{s}$ as given in (21), and the reserve cost $C_{r}$ as given in (22):

$$
\begin{aligned}
C_{o m}[k] & =\left[K_{o m, s} \cdot\left(P_{s}^{c}[k]+P_{s}^{d i s c}[k]\right)+\sum_{i=1}^{N_{g}} K_{o m, g} \cdot P_{g, i}[k]\right] \cdot \Delta t, \\
C_{g}[k]= & \sum_{i=1}^{N_{g}} K_{g} \cdot P_{g, i}[k] \cdot \Delta t, \\
C_{s}[k]= & \sum_{i=1}^{N_{g}}\left(S U_{i}[k]+S D_{i}[k]\right), \\
C_{r}[k]= & K_{r, s} \cdot\left(P_{s}^{u r}[k]+P_{s}^{d r}[k]\right)+\sum_{i=1}^{N_{g}} K_{r, g} \cdot P_{g, i}^{u r}[k]+K_{r, e u r} \cdot\left(\Delta P_{e}^{\max _{1}}[k]-P_{s}^{u r}[k]\right)+ \\
& K_{r, e d r} \cdot\left(\Delta P_{e}^{\max _{2}}[k]-P_{s}^{d r}[k]-\sum_{i=1}^{N_{g}} P_{g, i}^{u r}[k]\right) .
\end{aligned}
$$

Here, $k$ is the discrete-time index, $t$ is the index for the current instance, $M$ is the length of prediction horizon, and $\Delta t$ is the time duration of each step. In the cost function (18), a discount factor $\alpha$ is introduced to serve as the weights for each step with $0<\alpha \leq 1$. It is mostly used to determine the length of the optimization horizon for infinite horizon problems in the literature (see e.g., $[38,39]$ ), but here it is introduced to adjust the priorities of steps so that the system pays more attention to the scheduling in the near future [35].

Equation (19) represents the operation and maintenance cost of the devices. $P_{s}^{c}$ and $P_{s}^{\text {disc }}$ are the total charging and discharging power of the energy storage units, $N_{g}$ is the number of dispatchable generators, and $P_{g, i}$ is the operating power of the $i$-th generator; $K_{o m, s}$ and $K_{o m, g}$ are the corresponding coefficients related to the operation and maintenance. Equation (20) is the purchasing expense of the consumed non-renewable energy resources such as fuel or gas and $K_{g}$ is the transformed price of the energy. Generally, it takes a certain amount of time and high costs to switch on or shut down the generators, and Equation (21) limits the number of startups and shutdowns and avoids frequent switching operations. $S U_{i}[k]$ and $S D_{i}[k]$ denote the startup and shutdown costs of the $i$ th generator at time $k$, respectively. Equation (22) is a penalty term for the reserves. $P_{s}^{u r}$ and $P_{s}^{d r}$ are reserve variables of the storage units we introduced, which represent upward reserve power for charging and downward reserve power for discharging, respectively; $P_{g, i}^{u r}$ is also an introduced reserve variable and it denotes upward reserve operating power of the $i$-th generator. They are the extra system capacity we reserve in advance, which cannot be used at the dispatch stage, but can be used to compensate for the variations at the operation stage. $\Delta P_{e}^{\max _{1}}$ and $\Delta P_{e}^{\max _{2}}$ are the maximum power error between the selected scenarios in optimization and the actual situation in the conditional best and worse cases, respectively. $K_{r, s}, K_{r, g}, K_{r, e u r}$, and $K_{r, e d r}$ are coefficients for the reserves, and these coefficients are not constants. They are sensitive to the system state and the trends of RES generation and load demand in the future so that the reserve level can be adjusted accordingly. The penalty term puts soft constraints on the reservation level and facilitates a good tradeoff between economical efficiency and system reliability. 
It is noted that the microgrid optimal dispatch problem is finally transformed into an MILP problem. Branch-and-cut supplemented by heuristics can be used to solve the MILP problem with reasonable computational complexity [58-60].

\subsection{Two-Stage Control Model}

There are two stages in the control model based on the MPC framework and the schematic diagram is shown in Figure 3.

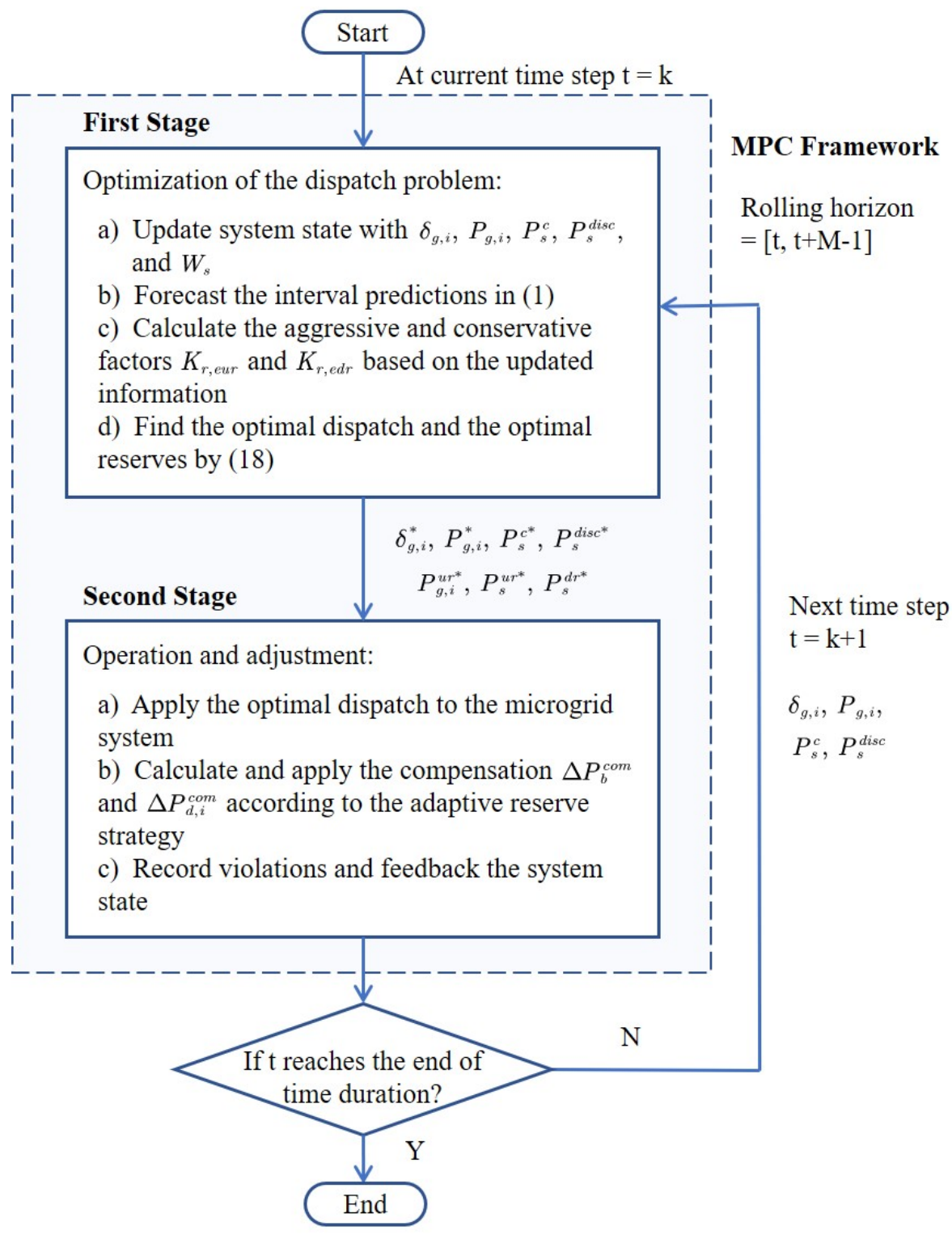

Figure 3. The schematic diagram of the two stage control model using the Model Predictive Control (MPC) framework and adaptive reserve strategy.

The first layer is the optimization stage, where the probabilistic optimization problem is solved to find the optimal scheduling as guidance for the actual operation. At this stage, system state is updated and the interval predictions for the next period are generated according to the feedback. Then the parameters $K_{r, \text { eur }}$ and $K_{r, e d r}$ in the reserve cost are modified based on the updated information, with which the aggressive and conservative level of the system changes. Finally, the reserve policy is adjusted and the optimal dispatch of the controllable components is obtained accordingly in a coupling manner.

The second one is the operation and adjustment stage. At this stage, the microgrid system first operates according to the control policy obtained at the optimization stage. 
In addition, variations are detected during the period and the system would respond to them using the reserved capacity on the basis of the adaptive reserve strategy. The primary control and the secondary control are generally employed here to modify the reference value and compensate for the differences [40,61]. System capacity is always limited and the load demand may be unsatisfied in terrible cases even the dispatch and the reserves are properly scheduled. Hence, when a violation occurs, the violated power and other corresponding information will be recorded for further analysis. At the end of the time interval, dynamic changes have been completed and the new system state is sent to the optimizer as feedback. Then the MPC horizon is shifted and a new dispatch and control problem is to be solved similarly.

\subsection{The Energy Management Strategy}

Figure 4 shows the flowchart of our method using the MPC approach, where some major processes of the adaptive reserve strategy are also displayed. In addition to the general operations of the MPC approach, the state of the system is also re-evaluated after the feedback so that the aggressive-conservative level is adjusted accordingly to regulate optimization and scheduling. Compensation operations are also added in the actual implementation.

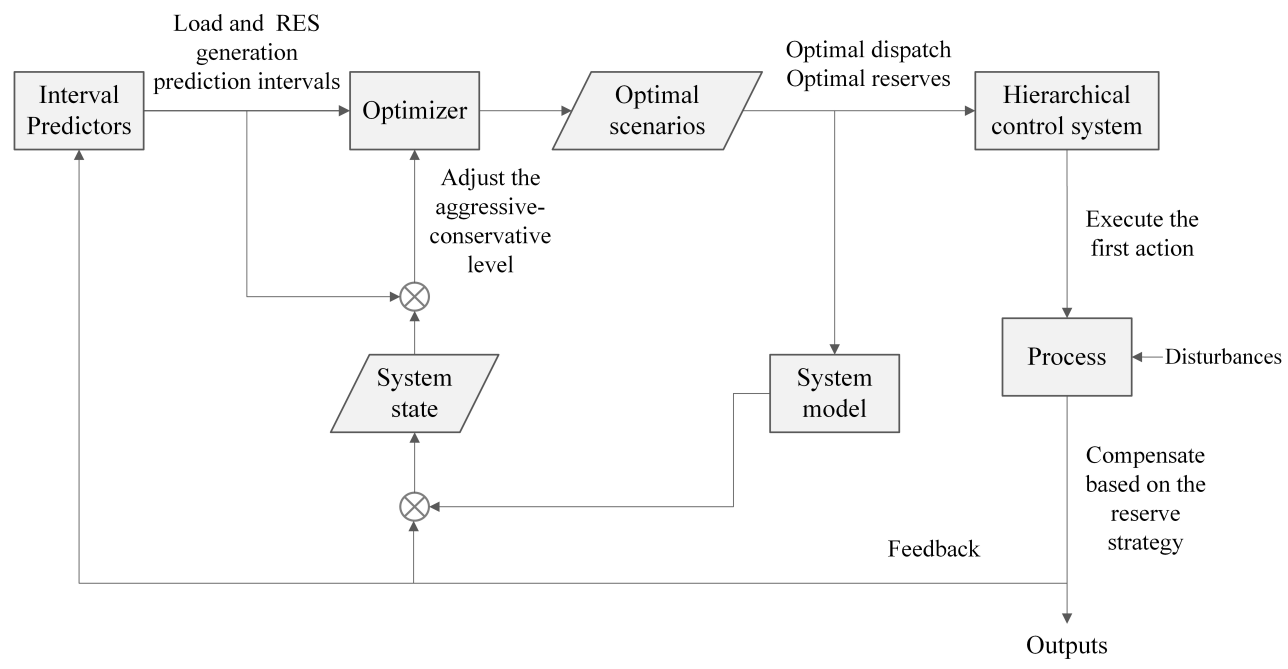

Figure 4. The flowchart of our method using the MPC approach as the framework.

Figure 5 displays the actual energy flow in the microgrid system and the signal flow in the energy management. In the energy flow, only the storage units could both provide and utilize the energy power. In the signal flow, the predictors first collect the historical actual data of RES generation and load demand at time step $k$, then predict the intervals in the future and send the prediction to the management center (MC). In the meantime, the dispatchable components also send the actual operations to the MC as feedback. Then based on the prediction and current system capacity, the MC could grasp the future trends and know how to schedule and prepare to maintain certain system capabilities. By solving a MILP problem, the optimal dispatch and proper reserves for the next time step are obtained in the $\mathrm{MC}$ and then transmitted to the corresponding devices. At the next time step $k+1$, the storage units and the dispatchable generators will operate referring to the optimal dispatch, and the actual power would be adjusted using the reserved capacity by the primary and secondary control to compensate for the potential variations. As a result, economic dispatches are achieved while ensuring system stability. 


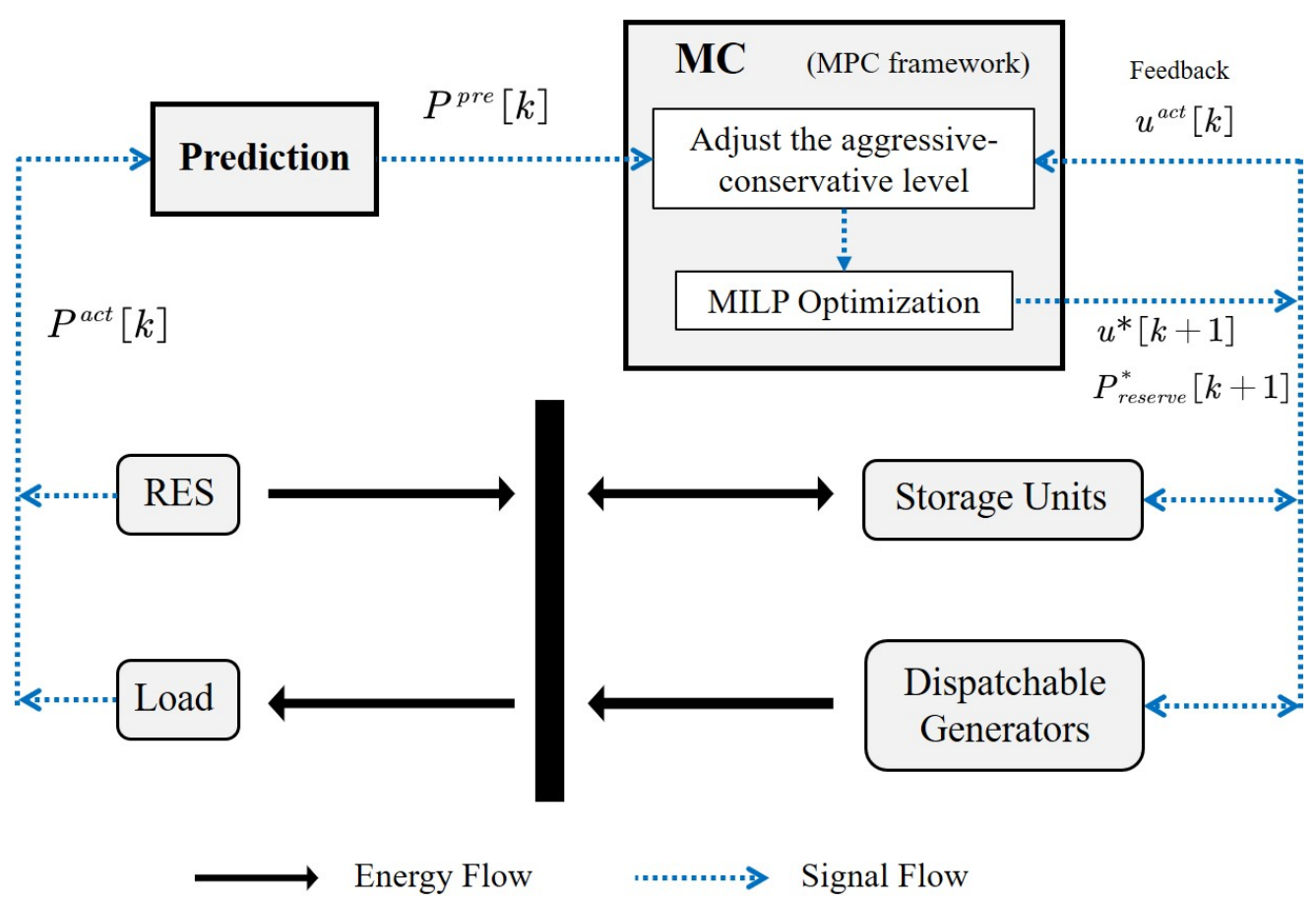

Figure 5. The energy flow and the signal flow in the system using our method.

\section{Simulations}

Case studies were implemented and simulated in MATLAB 2017b, and the optimization problem was modeled using YALMIP (Version 25 April 2019) [62] and solved using Gurobi 8.1 [63]. The program was run on a Super Server 4028GR-TR2 using two Intel(R) Xeon(R) E5-2698v4 2.20 GHz processors with 16 GB of RAM memory in Shenzhen, China.

The considered isolated microgrid in simulations is shown in Figure 2, consisting of a photovoltaic generation system, a wind turbine system, three sets of diesel generators, and one battery storage system. The values of the corresponding device parameters are selected the same as in $[17,64]$ and shown in Table 1 . The maximum operating power of the battery storage units is $150 \mathrm{~kW}$ with a capacity of $200 \mathrm{kWh}$. The rated power and the operating cost of the three diesel generators are set differently, so that the preference of the control strategy under different circumstances could be analyzed. The duration of the optimal dispatch problem is $24 \mathrm{~h}$ as most work sets, and the discrete-time step $\Delta t$ is $15 \mathrm{~min}$ [65]. MPC framework is utilized in the optimization and the rolling horizon $M$ is set to $24 \mathrm{~h}$. Thus, the total operation steps $N$ of the one-day control problem is 96 .

Table 1. Values of the parameters.

\begin{tabular}{cccc}
\hline Parameters & Value & Parameters & Value \\
\hline$W_{s}^{\max }$ & $200 \mathrm{kWh}$ & $N_{g}$ & 3 \\
$W_{s}^{\min }$ & $10 \mathrm{kWh}$ & $P_{g}^{\max }, i=1, \ldots, N_{g}$ & $20 / 40 / 60 \mathrm{~kW}$ \\
$P_{s}^{\max }$ & $150 \mathrm{~kW}$ & $K_{i}^{S U^{\prime}}, K_{i}^{S D}, i=1, \ldots, N_{g}$ & $5 / 10 / 15$ \\
$P_{s}^{\min }$ & 0 & $R^{\max }$ & $8 \mathrm{~kW}$ \\
$\eta$ & 0.9 & $R^{\min }$ & 0 \\
$\sigma_{s}$ & 0.05 & $\alpha$ & 0.8 \\
\hline
\end{tabular}

To verify the performance of our proposed method for the energy exchange control of isolated microgrids, simulations in the case of volatile renewable generation and uncertain prediction accuracy over the $24 \mathrm{~h}$ are conducted. Both the economical performance and the stability performance are discussed compared with the robust method and our previously proposed method. In addition, some performance metrics suitable for isolated microgrids are also introduced in this section. 


\subsection{Performance Metrics}

Comparisons are conducted to analyze system performance using different methods in terms of economy and reliability. The performance metrics we used are presented here.

\section{A. Economical Performance}

The economical performance of the microgrid system can be directly evaluated using the actual operation cost given by:

$$
F_{c}=\sum_{t=1}^{N}\left[C_{o m}[t]+C_{g}[t]+C_{s}[t]\right]
$$

where the extra penalty term is removed and the three costs included are calculated using Equations (19)-(21) with the corresponding operating power at the current time $t$.

\section{B. Reliability Performance}

The system reliability of isolated microgrids is evaluated from the three aspects of occurrence, interruption duration, and violated power. On the basis of [66], the following two metrics are employed to measure the system performance in this paper:

(1) Isolated Loss of Load Probability (ILOLP). This metric indicates the stability performance in terms of running time. It is the fraction of time duration that the load demand is not satisfied in isolated microgrids with:

$$
I L O L P=\frac{\text { Time duration that violations occur }}{\text { Total operation duration }}=\frac{n^{v i o} \Delta t}{T},
$$

where $n^{\text {vio }}$ is the number of violations, $\Delta t$ is the time interval of each step, and $T$ is the total operation duration;

(2) Isolated Load Loss Rate (ILLR). This metric describes the severity of the violations during the whole operation in isolated microgrids compared with the average load demand, which is formulated as:

$$
I L L R=\frac{\text { Average load loss }}{\text { Average load demand }}=\frac{I A L L}{\frac{1}{N} \sum_{i=1}^{N} P_{\text {load }}^{\text {act }}},
$$

where $N$ is the total operation steps and $P_{\text {load }}^{a c t}$ denotes the actual load demand power. IALL is a common metric called Isolated Average Load Loss presenting the unsatisfied load demand each time the violation occurs, and it is given by:

$$
I A L L=\frac{\text { Total violated energy }}{\text { Total number of violations }}=\frac{\frac{1}{N} \sum_{i=1}^{N} P^{v i o}}{n^{v i o}}
$$

with $P^{\text {vio }}$ denoting the violated load power.

\subsection{Case Study}

In this paper, we focus on improving our previous proposed method to increase the system's stability while maintaining the economic efficiency of isolated microgrids when the RES is highly volatile and the prediction accuracy is not always guaranteed. Simulations are conducted using our proposed method, our previous method, and the robust method. Table 2 shows the simulation results for the one-day energy exchange optimization using different methods. 
Table 2. Simulation results.

\begin{tabular}{cccc}
\hline Method & Number of Violations & Violated Power (kW) & Operation Cost (\$) \\
\hline Our proposed method & 0 & 0 & 1220.3 \\
Our previous method & 2 & 28.76 & 1392.2 \\
Existing robust method & 1 & 23.21 & 1513.0 \\
\hline
\end{tabular}

In terms of the system's stability and reliability, it can be seen that even the robust method may not be able to satisfy the entire load demand when the situation is hostile. Our previous proposed method also performs poorly and causes inevitable violations. However, our improved method successfully handles the issue and violations are avoided in this case. As for economic efficiency, we can see that our proposed method reduces the operation cost by about $12 \%$ compared with our previous method. It also reduces the cost by more than $19 \%$ when compared with the robust method.

To further analyze the effect of the improvements of the proposed method, the operation results of our proposed method are shown in Figure 6. The three dashed curves are actual power data of load, photovoltaic generation, and wind turbine generation, and the four solid curves are actual operation power of diesel generators and the storage units. It can be seen that the system tends to use the storage units and avoid the usage of generators whenever possible to save operation cost. It should also be noted that the three generators own different rated power and operating costs, and from the figure we can observe that the priorities of these generators vary in different scenarios. When the RES generation is far more larger than the load demand in the future, the system is more likely to use the smaller generator which has a lower startup and shutdown cost (see steps 27-37), since it holds the opinion that we do not need to prepare more capacity for the future so that we can save cost by using a smaller but cheaper generator. When more power is needed to supply for the demand in the future, the system tends to use the larger generator more so that it could compensate for more power differences and avoid the increased cost due to frequent shutdown and startup operations (see steps 83-96).

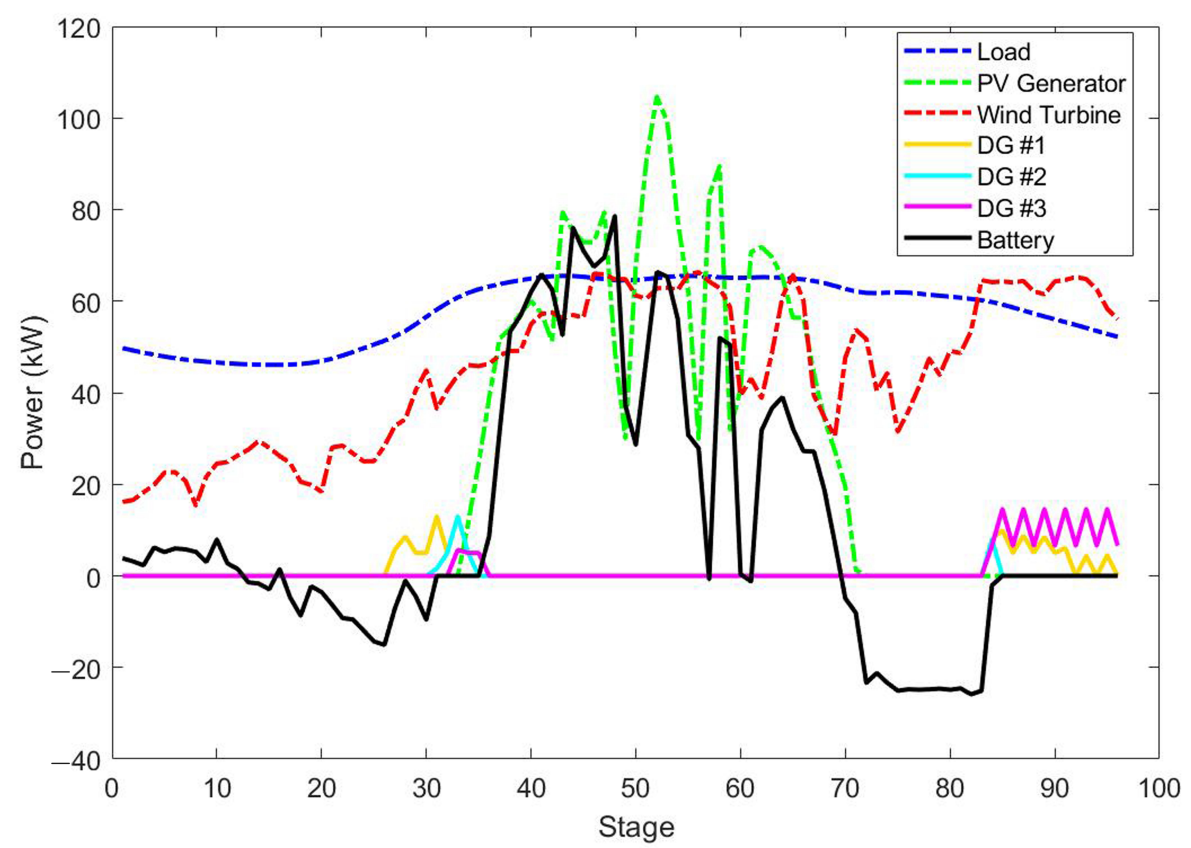

Figure 6. Actual power data of load and Renewable Energy Sources (RES) generation, and the actual operation results of diesel generator and energy storage units using our improved method. 
The operation power of all the diesel generators and energy storage units using the original and the new methods are shown in Figure 7. Two violations are recorded at time steps 86 and 88 for our previous method, and the corresponding violated load power are $6.87 \mathrm{~kW}$ and $21.89 \mathrm{~kW}$. From the figure, it is observed that the scheduling policies of the two methods are identical for most of the time, but they are different around the stages that are prone to cause violations. We can see that the new method prepares better and generates more power in advance around time step 85. It also reduces the number of devices running and smooths the operating power of generators to cut down the unnecessary startup and shutdown costs, smoothening the operating power. These demonstrate that the improved method is more aware of the system capacity and better monitors its trend in the near future, and in turn, it can better schedule so as to avoid violations and reduce cost.

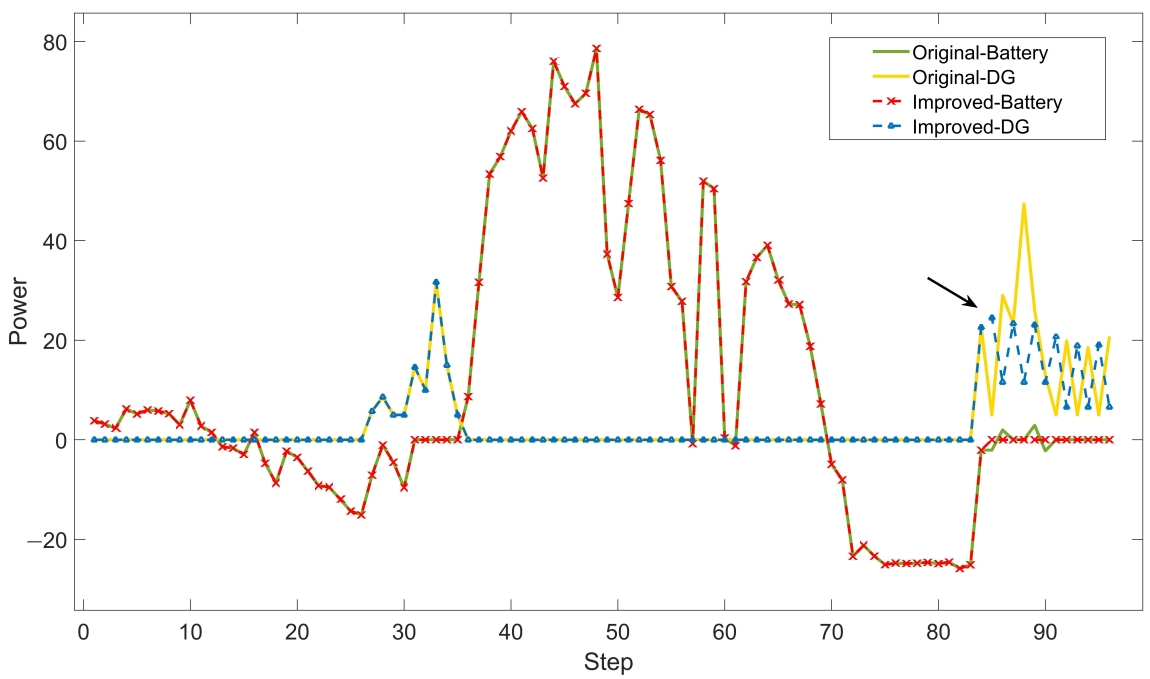

Figure 7. Actual operating power of diesel generators and energy storage units using the improved method and the previous method.

Figure 8 shows some of the simulation results that are related to the reserve strategy. It can be seen that the values of reserve variables vary with situations. The minimum and maximum values of the total reserve are about $2.77 \mathrm{~kW}$ and $26.19 \mathrm{~kW}$, which are about $4.1 \%$ and $38.8 \%$ of the average power of RES generation in the simulation. From the figure, significant reductions can be observed around the steps where RES generation could almost meet the load demand. This indicates the system has a good knowledge about the system capability and properly adjust the level of capability for various situations using the adaptive reserve strategy. The reserve level is lower when the situation is good, and it becomes higher when the differences between RES generation and load is large and system capacity is poor. These show the effort of our method to ensure system stability and save costs if possible. 


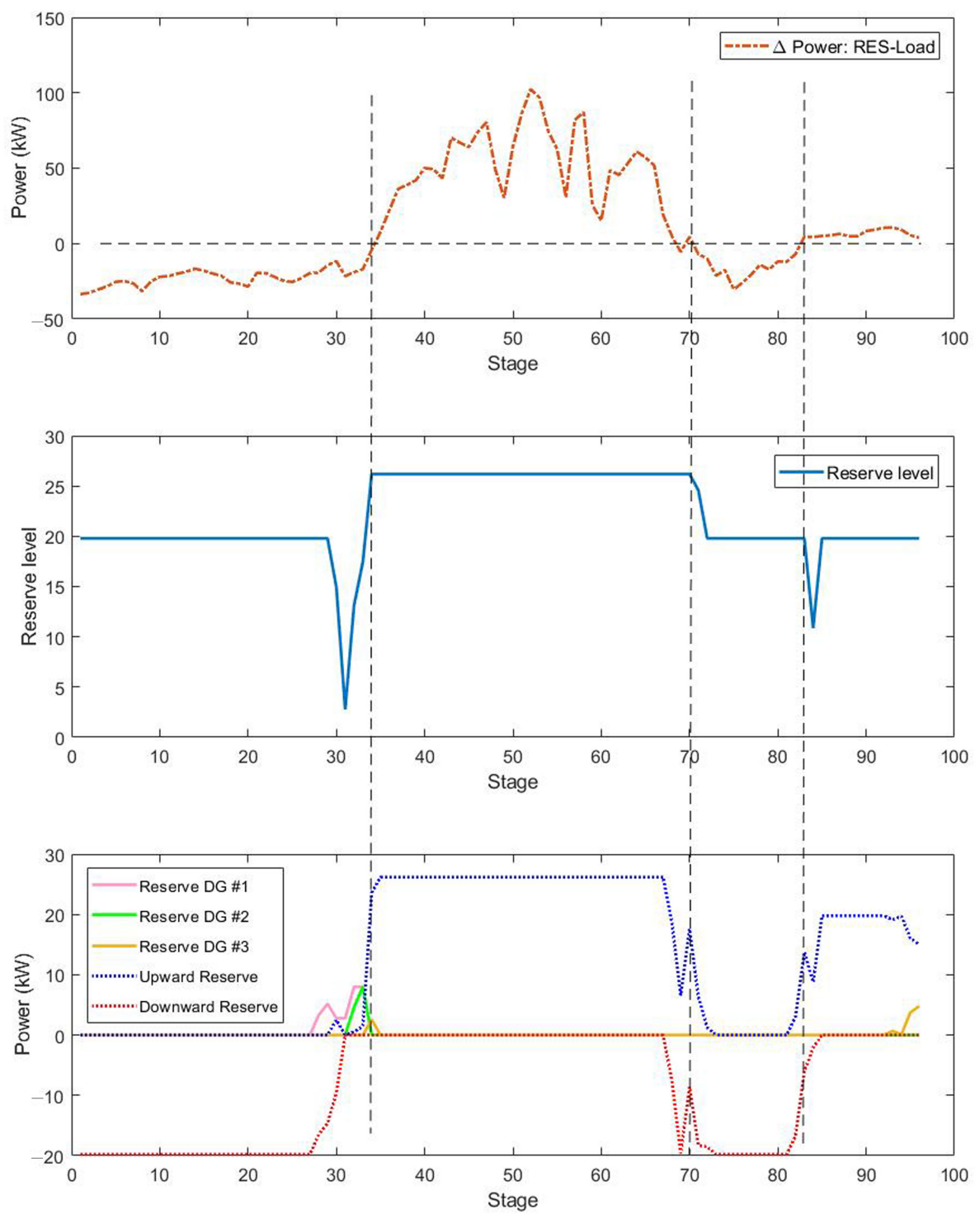

Figure 8. Simulation results related to the reserve strategy. (Top) the power differences of RES generation and load demand. (Middle) the total reserve level over stages. (Bottom) the reserve power in battery and DGs.

\subsection{Performance Analysis}

To diversify and complicate the cases of inaccurate predictions, we introduced some random variations into the actual data of RES generation and load demand and then repeat the entire simulations with the new data many times. The average performances in terms of economic efficiency and system reliability using different methods are shown in Table 3. The performance metrics presented in Section 5.1 are employed.

Table 3. Average performances of different methods.

\begin{tabular}{ccccc}
\hline Method & ILOLP (\%) & IALL (kW) & ILLR (\%) & Operation Cost (\$) \\
\hline Our proposed method & 0.52 & 13.86 & 23.96 & 1316.5 \\
Our previous method & 1.98 & 18.15 & 31.37 & 1400.9 \\
Existing robust method & 0.52 & 20.98 & 36.27 & 1434.0 \\
\hline
\end{tabular}


ILOLP indicates the proportion of violation duration to the total one-day optimization. It can be seen that the ILOLP of our previous method is much larger than that of the robust method, which means the probability of violation occurring is larger with the former method. However, when it comes to the IALL and ILLR, the situations are reversed. IALL describes the average violated power each time the violation occurs and ILLR shows the seriousness of the violations based on the average load demand. It can be observed that our previous method performs better than the robust method with less violated power and load shedding per time. Remarkably, the improved method we propose in this paper works well on both counts. It reduces $1.42 \%$ possibility in leading to violations when solving the probabilistic microgrid optimal dispatch problem compared with our previous method. It also cuts the violated power by about a third, and has a $7.41 \%$ reduction compared with the previous method and a $12.31 \%$ reduction compared with the robust method in ILLR. All in all, the proposed method performs the best in terms of both frequency and severity level of the violation and it could significantly increase the system's reliability. It has better capability to dispatch and control the microgrid system and satisfy the load demand, and even when the violation occurs inevitably, the degree of negative influence is also lighter.

As for the economical performance, our proposed method also works well. It reduces $6.02 \%$ and $8.19 \%$ operation cost compared with the previous method and the robust method, respectively. It is noted that our approach not only maintains the original economic benefits but even further reduces the cost.

Therefore, we can draw the conclusion that our proposed method achieves the best stability and economic performances. It can make good use of the interval predictions and well handle the probabilistic dispatch problem of isolated microgrids with a high penetration of intermittent RES.

\subsection{The Extreme Case Study}

Note that the aim of this paper is to improve the system stability of island microgrids especially when the RES is too volatile to predict and handle. It is generally acknowledged that RES of high volatility greatly increases the difficulty of forecasting and affects the prediction accuracy, which may lead to unaffordable consumption and even severe system failures. To further study the effect of our proposed method, optimization with no prediction inputs is also taken into consideration in the simulations which is an extreme case. Although this case is almost impossible to occur in reality, it is worth studying because it can verify the effectiveness of the control method under the worst case.

Table 4 shows the results of the performance metrics in the simulation in the extreme case, where predictions are not provided and our method optimizes and controls the system by assuming the RES generation and load demand power are zero. By comparing the results in Table 3, it can be observed that the probability and the degree of violations have increased since the optimizer does not know the trends and cannot prepare in advance for future variations. However, the performance deterioration is not significant, and the operation cost does not increase much. This indicates the positive effect of the adaptive reserve strategy in our method for guaranteeing the system stability. Although the predictions are not available, the system could still analyze the system state and adjust the aggressiveconservative level accordingly. Besides, the optimization still works with reserves properly determined so that variations can be compensated to some extent based on the reserve strategy at the operation stage.

Table 4. Performances of our methods in an extreme case.

\begin{tabular}{ccccc}
\hline Method & ILOLP (\%) & IALL (kW) & ILLR (\%) & Operation Cost (\$) \\
\hline Our method with no prediction inputs & 2.08 & 24.07 & 41.61 & 1578.05 \\
Only hierarchical control with no prediction inputs & 53.13 & 39.21 & 67.78 & 10109.09 \\
\hline
\end{tabular}


In addition, the simulation that only MC is used are also conducted here to highlight the function of the optimization in our method. The optimizer is removed in the simulation and the microgrid system only responds to the demand based on the predetermined response strategy with the general hierarchical control model. It can be seen that the stability and economical performances are really poor in this case. The possibility of violations increases dramatically and most of the load demand has to be shed. Worse still, the operation cost is increased by $540.6 \%$ compared with our method. The huge differences in the performances demonstrate the effectiveness of the optimization and the reserve strategy in our approach. Our proposed method can not only utilize the information of interval predictions, but also effectively deal with RES with high fluctuation, maintaining certain system stability even under the most extreme cases.

\section{Conclusions}

In this paper, an adaptive probabilistic energy management method was proposed to further improve system stability while ensuring the economical efficiency of isolated microgrids with interval predictors. MPC was employed as the optimization framework with the discount factor adjusting the step weights, where scenario-selected optimizer and adaptive reserve strategy were used in combination to make good use of the interval predictions and achieve stability and economy efficiency. Appropriate scenarios were selected from the predictions according to the evaluation of future trends and system capacity, and the adaptive reserve strategy made further use of the underlying information of predictions to prepare more for possible fluctuations in the future. The aggressive and conservative level of the system was also introduced to guarantee certain system capability and adjust the tendency of the system so that the system stability was ensured when the situation is hostile and cost-effectiveness was achieved when the situation was better. The two-stage control model was presented to assist the implementation of the reserve strategy in optimization and operation. Finally, the microgrid optimal dispatch issue was formulated to an MILP problem which could be solved within a reasonable time.

Case studies were conducted to validate the performance of the proposed method in terms of system stability and economic efficiency. Results showed that our method better handled the isolated microgrid optimal dispatch problem compared with other methods, effectively improving the system stability and reducing the total cost. Moreover, simulations using our proposed method in extreme case were also studied, which showed that our method was capable of coping with the dramatic volatility of RES and load to guarantee the robustness of isolated microgrids.

It is noted that our proposed method could give good microgrid dispatch solutions with low requirement for the prediction accuracy, which shows its potential in handling the situations where prediction accuracy is limited. In future, we could extend the method to solve the energy exchange problem with disturbances caused by sensor failures, information loss in communication, etc. In addition, more complicated systems such as those with thermal loads and corresponding components can also be studied using our proposed methods.

Author Contributions: Conceptualization, D.D. and L.Y.; methodology, J.C.; software, J.C.; validation, J.C. and D.D.; formal analysis, J.C. and D.D.; investigation, J.C. and D.D.; resources, L.Y. and X.C.; writing—original draft preparation, J.C.; writing—review and editing, D.D., X.C., L.Y. and S.C.; supervision, L.Y. and S.C.; project administration, D.D. and X.C.; funding acquisition, L.Y. and S.C. All authors have read and agreed to the published version of the manuscript.

Funding: This work was supported in part by Key-Area Research and Development Program of Guangdong Province Project under Grant No. 2018B030338001, Natural Science Foundation of China under Grant NSFC-61629101, Shenzhen Fundamental Research Fund under Grant No. JCYJ20170411102217994, the National Science Foundation under Grant DMS-1923142, the Open Research Fund from Shenzhen Research Institute of Big Data under Grant 2019ORF01006, the National Key R\&D Program of China under Grant No. 2018YFB1800800, and Guangdong Zhujiang Project under Grant No. 2017ZT07X152. 
Informed Consent Statement: Informed consent was obtained from all subjects involved in the study.

Data Availability Statement: The data presented in this study are available on request from the corresponding author. The data are not publicly available due to privacy restrictions.

Conflicts of Interest: The authors declare no conflict of interest.

\section{References}

1. Al-Turjman, F.; Abujubbeh, M. IoT-enabled smart grid via SM: An overview. Future Gener. Comput. Syst. 2019, 96, 579-590. [CrossRef]

2. Dragičević, T.; Siano, P.; Prabaharan, S. Future generation 5G wireless networks for smart grid: A comprehensive review. Energies 2019, 12, 2140

3. Hui, H.; Ding, Y.; Shi, Q.; Li, F.; Song, Y.; Yan, J. 5G network-based Internet of Things for demand response in smart grid: A survey on application potential. Appl. Energy 2020, 257, 113972. [CrossRef]

4. Lasseter, R.H.; Paigi, P. Microgrid: A conceptual solution. In Proceedings of the 2004 IEEE 35th Annual Power Electronics Specialists Conference (IEEE Cat. No.04CH37551), Aachen, Germany, 20-25 June 2004; Volume 6, pp. 4285-4290.

5. Kazerani, M.; Tehrani, K. Grid of Hybrid AC/DC Microgrids: A New Paradigm for Smart City of Tomorrow. In Proceedings of the 2020 IEEE 15th International Conference of System of Systems Engineering (SoSE), Budapest, Hungary, 2-4 June 2020; pp. 175-180.

6. Badal, F.R.; Das, P.; Sarker, S.K.; Das, S.K. A survey on control issues in renewable energy integration and microgrid. Prot. Control Mod. Power Syst. 2019, 4, 8. [CrossRef]

7. Pedrasa, M.A.; Spooner, T. A survey of techniques used to control microgrid generation and storage during island operation. In Proceedings of the 2006 Australasian Universities Power Engineering Conference (AUPEC'06), Melbourne, Australia, 10-13 December 2006; pp. 1-6.

8. Olivares, D.E.; Cañizares, C.A.; Kazerani, M. A Centralized Energy Management System for Isolated Microgrids. IEEE Trans. Smart Grid 2014, 5, 1864-1875. [CrossRef]

9. Kayalvizhi, S.; Vinod Kumar, D.M. Load Frequency Control of an Isolated Micro Grid Using Fuzzy Adaptive Model Predictive Control. IEEE Access 2017, 5, 16241-16251. [CrossRef]

10. Sobu, A.; Wu, G. Optimal operation planning method for isolated micro grid considering uncertainties of renewable power generations and load demand. In Proceedings of the IEEE PES Innovative Smart Grid Technologies, Washington, DC, USA, 16-20 January 2012; pp. 1-6.

11. Wan, C.; Zhao, J.; Song, Y.; Xu, Z.; Lin, J.; Hu, Z. Photovoltaic and solar power forecasting for smart grid energy management. CSEE J. Power Energy Syst. 2015, 1, 38-46. [CrossRef]

12. Soares, J.; Pinto, T.; Lezama, F.; Morais, H. Survey on complex optimization and simulation for the new power systems paradigm. Complexity 2018, 2018, 2340628. [CrossRef]

13. Katiraei, F.; Iravani, R.; Hatziargyriou, N.; Dimeas, A. Microgrids management. IEEE Power Energy Mag. 2008, 6, 54-65. [CrossRef]

14. Sortomme, E.; El-Sharkawi, M.A. Optimal Power Flow for a System of Microgrids with Controllable Loads and Battery Storage. In Proceedings of the 2009 IEEE/PES Power Systems Conference and Exposition, Seattle, WA, USA , 15-18 March 2009; pp. 1-5.

15. Sachs, J.; Sawodny, O. A Two-Stage Model Predictive Control Strategy for Economic Diesel-PV-Battery Island Microgrid Operation in Rural Areas. IEEE Trans. Sustain. Energy 2016, 7, 903-913. [CrossRef]

16. Luna, A.C.; Diaz, N.L.; Graells, M.; Vasquez, J.C.; Guerrero, J.M. Mixed-Integer-Linear-Programming-Based Energy Management System for Hybrid PV-Wind-Battery Microgrids: Modeling, Design, and Experimental Verification. IEEE Trans. Power Electron. 2017, 32, 2769-2783. [CrossRef]

17. Parisio, A.; Rikos, E.; Glielmo, L. A Model Predictive Control Approach to Microgrid Operation Optimization. IEEE Trans. Control. Syst. Technol. 2014, 22, 1813-1827. [CrossRef]

18. Nwulu, N.I.; Xia, X. Optimal dispatch for a microgrid incorporating renewables and demand response. Renew. Energy 2017, 101, 16-28. [CrossRef]

19. Chaudhary, P.; Rizwan, M. Energy management supporting high penetration of solar photovoltaic generation for smart grid using solar forecasts and pumped hydro storage system. Renew. Energy 2018, 118, 928-946. [CrossRef]

20. Gil-González, W.; Montoya, O.D.; Holguín, E.; Garces, A.; Grisales-Noreña, L.F. Economic dispatch of energy storage systems in dc microgrids employing a semidefinite programming model. J. Energy Storage 2019, 21, 1-8. [CrossRef]

21. Nasr, M.A.; Nikkhah, S.; Gharehpetian, G.B.; Nasr-Azadani, E.; Hosseinian, S.H. A multi-objective voltage stability constrained energy management system for isolated microgrids. Int. J. Electr. Power Energy Syst. 2020, 117, 105646. [CrossRef]

22. Pinson, P.; Kariniotakis, G. Conditional Prediction Intervals of Wind Power Generation. IEEE Trans. Power Syst. 2010, 25, 1845-1856. [CrossRef]

23. Sobri, S.; Koohi-Kamali, S.; Rahim, N.A. Solar photovoltaic generation forecasting methods: A review. Energy Convers. Manag. 2018, 156, 459-497. [CrossRef] 
24. Verrilli, F.; Parisio, A.; Glielmo, L. Stochastic model predictive control for optimal energy management of district heating power plants. In Proceedings of the 2016 IEEE 55th Conference on Decision and Control (CDC), Las Vegas, NV, USA, 12-14 December 2016; pp. 807-812.

25. Olivares, D.E.; Lara, J.D.; Cañizares, C.A.; Kazerani, M. Stochastic-Predictive Energy Management System for Isolated Microgrids. IEEE Trans. Smart Grid 2015, 6, 2681-2693. [CrossRef]

26. Rastegar, M.; Fotuhi-Firuzabad, M.; Zareipour, H.; Moeini-Aghtaieh, M. A Probabilistic Energy Management Scheme for Renewable-Based Residential Energy Hubs. IEEE Trans. Smart Grid 2017, 8, 2217-2227. [CrossRef]

27. Ma, X.; Dong, J.; Djouadi, S.M.; Nutaro, J.J.; Kuruganti, T. Stochastic control of energy efficient buildings: A semidefinite programming approach. In Proceedings of the 2015 IEEE International Conference on Smart Grid Communications (SmartGridComm), Miami, FL, USA, 2-5 November 2015; pp. 780-785.

28. Kou, P.; Liang, D.; Gao, L.; Gao, F. Stochastic Coordination of Plug-In Electric Vehicles and Wind Turbines in Microgrid: A Model Predictive Control Approach. IEEE Trans. Smart Grid 2016, 7, 1537-1551. [CrossRef]

29. Sáez, D.; Ávila, F.; Olivares, D.; Cañizares, C.; Marín, L. Fuzzy Prediction Interval Models for Forecasting Renewable Resources and Loads in Microgrids. IEEE Trans. Smart Grid 2015, 6, 548-556. [CrossRef]

30. Pekaslan, D.; Wagner, C.; Garibaldi, J.M.; Marin, L.G.; Sáez, D. Uncertainty-Aware Forecasting of Renewable Energy Sources. In Proceedings of the 2020 IEEE International Conference on Big Data and Smart Computing (BigComp), Busan, Korea, 19-22 Febuary 2020; pp. 240-246.

31. Marín, L.G.; Cruz, N.; Sáez, D.; Sumner, M.; Núñez, A. Prediction interval methodology based on fuzzy numbers and its extension to fuzzy systems and neural networks. Expert Syst. Appl. 2019, 119, 128-141. [CrossRef]

32. Valencia, F.; Sáez, D.; Collado, J.; Ávila, F.; Marquez, A.; Espinosa, J.J. Robust Energy Management System Based on Interval Fuzzy Models. IEEE Trans. Control Syst. Technol. 2016, 24, 140-157. [CrossRef]

33. Cartagena, O.; Muñoz-Carpintero, D.; Sáez, D. A Robust Predictive Control Strategy for Building HVAC Systems Based on Interval Fuzzy Models. In Proceedings of the 2018 IEEE International Conference on Fuzzy Systems (FUZZ-IEEE), Rio de Janeiro, Brazil, 8-13 July 2018; pp. 1-8.

34. Mohan, V.; Suresh, R.; Singh, J.G.; Ongsakul, W.; Madhu, N. Microgrid Energy Management Combining Sensitivities, Interval and Probabilistic Uncertainties of Renewable Generation and Loads. IEEE J. Emerg. Sel. Top. Circuits Syst. 2017, 7, 262-270. [CrossRef]

35. Cheng, J.; Duan, D.; Cheng, X.; Yang, L.; Cui, S. Probabilistic Microgrid Energy Management with Interval Predictions. Energies 2020, 13, 3116. [CrossRef]

36. Vandoorn, D.Q.; Rawlings, J.B.; Rao, C.V.; Scokaert, P.O.M. Constrained model predictive control: Optimality and stability. Automatica 2000, 36, 789-814.

37. Palma-Behnke, R.; Benavides, C.; Lanas, F.; Severino, B.; Reyes, L.; Llanos, J.; Sáez, D. A Microgrid Energy Management System Based on the Rolling Horizon Strategy. IEEE Trans. Smart Grid 2013, 4, 996-1006. [CrossRef]

38. Bertsekas, D.P. Dynamic Programming and Optimal Control; Athena Scientific: Nashua, NH, USA, $1995 ;$ pp. $402-445$.

39. Venayagamoorthy, G.K.; Sharma, R.K.; Gautam, P.K.; Ahmadi, A. Dynamic Energy Management System for a Smart Microgrid. IEEE Trans. Neural Netw. Learn. Syst. 2016, 1, 1643-1656. [CrossRef]

40. Bidram, A.; Davoudi, A. Hierarchical Structure of Microgrids Control System. IEEE Trans. Smart Grid 2012, 3, 1963-1976. [CrossRef]

41. Zhang, Y.; Gatsis, N.; Giannakis, G.B. Robust Energy Management for Microgrids With High-Penetration Renewables. IEEE Trans. Sustain. Energy 2013, 4, 944-953. [CrossRef]

42. Leonori, S.; Paschero, M.; Mascioli, F.M.F.; Rizzi, A. Optimization strategies for Microgrid energy management systems by Genetic Algorithms. Appl. Soft Comput. 2020, 86, 105903. [CrossRef]

43. Tehrani, K.; Member, I. A Smart Cyber Physical Multi-Source Energy System for an Electric Vehicle Prototype. J. Syst. Archit. 2020, 111, 101804. [CrossRef]

44. Griva, I.; Nash, S.G.; Sofer, A. Linear and Nonlinear Optimization; SIAM: Philadelphia, PA, USA, 2009; pp. 149-162.

45. Tsikalakis, A.G.; Hatziargyriou, N.D. Centralized control for optimizing microgrids operation. In Proceedings of the 2011 IEEE Power and Energy Society General Meeting, Detroit, MI, USA, 24-28 July 2011; pp. 1-8.

46. Paul, T.G.; Hossain, S.J.; Ghosh, S.; Mandal, P.; Kamalasadan, S. A Quadratic Programming Based Optimal Power and Battery Dispatch for Grid-Connected Microgrid. IEEE Trans. Ind. Appl. 2018, 54, 1793-1805. [CrossRef]

47. An, L.N.; Quoc-Tuan, T. Optimal energy management for grid connected microgrid by using dynamic programming method. In Proceedings of the 2015 IEEE Power Energy Society General Meeting, Denver, CO, USA, 26-30 July 2015; pp. 1-5.

48. Kanchev, H.; Lu, D.; Colas, F.; Lazarov, V.; Francois, B. Energy Management and Operational Planning of a Microgrid With a PV-Based Active Generator for Smart Grid Applications. IEEE Trans. Ind. Electron. 2011, 58, 4583-4592. [CrossRef]

49. Elsied, M.; Oukaour, A.; Youssef, T.; Gualous, H.; Mohammed, O. An advanced real time energy management system for microgrids. Energy 2016, 114, 742-752. [CrossRef]

50. Hossain, M.A.; Pota, H.R.; Squartini, S.; Abdou, A.F. Modified PSO algorithm for real-time energy management in grid-connected microgrids. Renew. Energy 2019, 136, 746-757. [CrossRef]

51. Ghorbani, S.; Rahmani, R.; Unland, R. Multi-agent autonomous decision making in smart micro-grids' energy management: A decentralized approach. In Proceedings of the German Conference on Multiagent System Technologies, Leipzig, Germany, 23-26 August 2017; Springer: Berlin/Heidelberg, Germany, 2017; pp. 223-237. 
52. Su, W.; Wang, J.; Roh, J. Stochastic energy scheduling in microgrids with intermittent renewable energy resources. IEEE Trans. Smart grid 2013, 5, 1876-1883. [CrossRef]

53. Xiang, Y.; Liu, J.; Liu, Y. Robust energy management of microgrid with uncertain renewable generation and load. IEEE Trans. Smart Grid 2015, 7, 1034-1043. [CrossRef]

54. Mondal, A.; Misra, S.; Patel, L.S.; Pal, S.K.; Obaidat, M.S. DEMANDS: Distributed energy management using noncooperative scheduling in smart grid. IEEE Syst. J. 2017, 12, 2645-2653. [CrossRef]

55. Kuznetsova, E.; Li, Y.F.; Ruiz, C.; Zio, E.; Ault, G.; Bell, K. Reinforcement learning for microgrid energy management. Energy 2013, 59, 133-146. [CrossRef]

56. Moehle, N.; Busseti, E.; Boyd, S.; Wytock, M. Dynamic energy management. arXiv 2019, arXiv:1903.06230.

57. Leonori, S.; Martino, A.; Mascioli, F.M.F.; Rizzi, A. Microgrid Energy Management Systems Design by Computational Intelligence Techniques. Appl. Energy 2020, 277, 115524. [CrossRef]

58. Richards, A.; How, J. Mixed-integer programming for control. In Proceedings of the 2005 American Control Conference, Portland, OR, USA, 8-10 June 2005; Volume 4, pp. 2676-2683.

59. Padberg, M.; Rinaldi, G. A branch-and-cut algorithm for the resolution of large-scale symmetric traveling salesman problems. SIAM Rev. 1991, 33, 60-100. [CrossRef]

60. Chen, Y.; Casto, A.; Wang, F.; Wang, Q.; Wang, X.; Wan, J. Improving Large Scale Day-Ahead Security Constrained Unit Commitment Performance. IEEE Trans. Power Syst. 2016, 31, 4732-4743. [CrossRef]

61. Morstyn, T.; Hredzak, B.; Agelidis, V.G. Control Strategies for Microgrids With Distributed Energy Storage Systems: An Overview. IEEE Trans. Smart Grid 2018, 9, 3652-3666. [CrossRef]

62. Löfberg, J. YALMIP: A toolbox for modeling and optimization in MATLAB. In Proceedings of the 2004 IEEE International Conference on Robotics and Automation, New Orleans, LA, USA, 26 April-1 May 2004; pp. 284-289.

63. Gurobi Optimization Inc. Gurobi Optimizer Reference Manual. 2014. Available online: http://www.gurobi.com (accessed on 22 December 2020 ).

64. Gu, W.; Wang, Z.; Wu, Z.; Luo, Z.; Tang, Y.; Wang, J. An Online Optimal Dispatch Schedule for CCHP Microgrids Based on Model Predictive Control. IEEE Trans. Smart Grid 2017, 8, 2332-2342. [CrossRef]

65. Raimondi Cominesi, S.; Farina, M.; Giulioni, L.; Picasso, B.; Scattolini, R. A Two-Layer Stochastic Model Predictive Control Scheme for Microgrids. IEEE Trans. Control Syst. Technol. 2018, 26, 1-13. [CrossRef]

66. Wang, S.; Li, Z.; Wu, L.; Shahidehpour, M.; Li, Z. New Metrics for Assessing the Reliability and Economics of Microgrids in Distribution System. IEEE Trans. Power Syst. 2013, 28, 2852-2861. [CrossRef] 